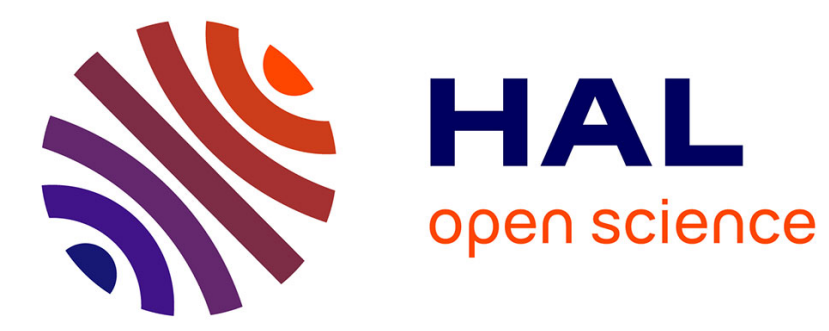

\title{
Comment transformer un produit en marchandise et lui attribuer un prix: le traitement de la viande dans la grande distribution
}

Guilhem Anzalone

\section{To cite this version:}

Guilhem Anzalone. Comment transformer un produit en marchandise et lui attribuer un prix: le traitement de la viande dans la grande distribution. Sociologie du Travail, 2009, 51 (1), pp.64-77. 10.1016/j.soctra.2008.12.004 . hal-01395318

\section{HAL Id: hal-01395318 \\ https://hal.science/hal-01395318}

Submitted on 10 Nov 2016

HAL is a multi-disciplinary open access archive for the deposit and dissemination of scientific research documents, whether they are published or not. The documents may come from teaching and research institutions in France or abroad, or from public or private research centers.
L'archive ouverte pluridisciplinaire HAL, est destinée au dépôt et à la diffusion de documents scientifiques de niveau recherche, publiés ou non, émanant des établissements d'enseignement et de recherche français ou étrangers, des laboratoires publics ou privés. 
Comment transformer un produit en marchandise et lui attribuer un prix :

le traitement de la viande dans la grande distribution.

Guilhem ANZALONE.

Centre de Sociologie des Organisations

19 rue Amélie,

75007 Paris.

Tel : 01.40.62.65.70.

Fax : 01.47.05.35.55.

g.anzalone@.cso.cnrs.fr

Centre Maurice Halbwachs, équipe ETT.

48 boulevard Jourdan,

75014 Paris. 


\section{Introduction.}

Si dans son célèbre ouvrage (1983) Karl Polanyi caractérise la terre, le travail et la monnaie comme des marchandises fictives puisqu'ils n'ont pas été produit pour être vendus sur un marché, cela ne doit pas laisser penser pour autant « que les autres biens présents sur le marché seraient quant à eux de plein droit des marchandises » (Vatin, 1996). Et de fait, de nombreux travaux se sont attachés à décrire les conditions dans lesquelles des biens et services peuvent constituer des marchandises, que ce soit dans les termes de l'anthropologie (Appadurai 1986, Testart 2001, Weber 2000), de l'économie des conventions (Eymard-Duvernay 1986), de la sociologie (Callon 1998, 2000 ; Chantelat 2002) et de l'histoire économique (Stanziani 2005, 2003). Le dossier débat organisé par Sociologie du travail (2002) a été l'occasion de confronter plusieurs de ces approches autour de la notion de qualité. Ce dossier et les contributions qu'il a plus ou moins directement suscitées (Stanziani 2003 bis ; Chiffoleau et Laporte 2004 ; Barrey 2006 ; Dubuisson-Quellier 2006) ont mis au premier plan les questions de l'identification et de l'évaluation des biens (y compris la formation de leurs prix), en soulignant d'ailleurs la difficulté de les traiter conjointement.

La commercialisation de la viande de bœuf par la grande distribution apparaît comme un terrain privilégié pour cette exploration, tant en raison des caractéristiques du produit que de celle de l'acteur ${ }^{1}$. Issue d'une chair animale hétérogène

\footnotetext{
${ }^{1}$ Ce texte s'appuie sur une enquête menée en 2005 dans un groupe de distribution gérant en franchise dix huit supermarchés et trois hypermarchés dans le Sud de la France, et dans un supermarché parisien appartenant à une autre enseigne. L'orientation du groupe est largement calquée sur celles des enseignes, de manière à bénéficier de leur travail ; il y a par exemple au sein d'une enseigne et pour chaque rayon des moniteurs techniques tant pour les magasins propres que pour les franchisés. Ce groupe n'a donc pas de moniteurs techniques propres, excepté le moniteur viande qui s'occupe de tout ce qui concerne les rayons boucherie et volaille des supermarchés. Cette organisation a comme particularité notable de concentrer sur une seule personne les relations avec les
} 
(d'une carcasse à une autre), complexe (chaque carcasse étant composée de différents muscles) et biologiquement instable, la viande doit passer par un certain nombre de transformations pour devenir une marchandise, et ces opérations sont d'autant plus stratégiques à observer que la grande distribution se caractérise par son degré et sa puissance de professionnalisation de l'activité marchande. L'intérêt est de centrer l'analyse sur un produit et sur sa circulation à un stade où l'organisation productive et le marché sont étroitement associés, la viande étant un des rares produits pour lesquels la grande distribution, entre les opérations d'achat et de vente constitutives de son métier, exécute elle-même et dans le cadre de ses magasins les dernières étapes du processus de production. Le choix de cette focale se justifie par la forte asymétrie qui existe entre les acteurs respectifs de l'offre et de la demande en termes de capacité de structuration de l'échange. Du fait de ses compétences techniques et de sa connaissances de l'amont et de l'aval, la grande distribution prend le pas sur des clients qu'elle canalise en partie en fonction de sa logique interne. Elle joue donc un rôle prépondérant dans la mise en forme et l'évaluation des produits, et pour cette raison l'amont de la filière et les pratiques de la grande distribution sont privilégiés dans ce texte. Cet angle de vue permet d'observer le traitement de la viande, en entendant par cette expression les transformations de la matière et, dans un sens analogue au traitement de données, le travail par lequel on en extrait des informations pour les exploiter : on entre ainsi dans le détail des mécanismes concrets de marchandisation, si l'on comprend par là non pas l'extension du domaine où s'exerce la loi du marché, mais plutôt l'en-

fournisseurs, le contrôle du travail en rayon, la fixation des prix et l'application de la politique commerciale. 
semble de procédés par lequel un produit est objectivé et apprécié de manière à pouvoir être échangé indépendamment des relations personnelles existant entre le vendeur et 1'acheteur (Testart 2001 ; Weber 2000 ; Eymard-Duvernay 1986). Pour ces mêmes raisons, c'est également un terrain propice à l'étude de la formation des prix : point central de la marchandisation, celle-ci est de plus en plus investie empiriquement par les sociologues, et notamment en ce qui concerne les procédures concrètes de formation des prix. C'est ce questionnement sur les mécanismes d'objectivation et d'appréciation (Eymard-Duvernay 1986, 2007 ; Weber 2000 ; Sociologie du travail 2002 ; Callon et Muniesa 2003 ; Barrey 2006) que voudrait prolonger cet article.

Aborder cette question à partir de la mise en forme d'un bien le long de sa trajectoire donne les moyens de décrire précisément les opérations de marchandisation à l'œuvre, ainsi que l'articulation construite par la grande distribution entre les processus d'identification et d'évaluation. En laissant hors de l'analyse les dispositifs spécifiquement destinés aux clients pour se centrer sur ceux qui sont mobilisés par la grande distribution, on peut montrer comment ce bien est objectivé le long de sa circulation (2.), et comment sa valorisation et l'élaboration de son prix s'appuient sur la mise en relation et le traitement des données issues de cette objectivation (3.). Autrement dit, l'évaluation des biens et services par la grande distribution résulte dans ce cas de la combinaison de données construites à partir de critères utilisés pour leur identification. 


\section{L'objectivation de la viande à l'épreuve de la transformation des carcasses.}

La viande devient une marchandise par un processus d'objectivation permettant de faire apparaître des caractéristiques à partir de l'hétérogénéité des carcasses, et ce de manière univoque pour les acteurs, quelles que soient les particularités de ceux-ci. Ce processus simultané d'homogénéisation et de différenciation se déroule avec la circulation de la viande le long de la filière, par la mobilisation d'instruments d'identification et d'évaluation tels que des dispositifs métrologiques, grilles de classification et autres nomenclatures, qui ont été progressivement mis en place (2.1.) et que l'on retrouve dans les transactions entre la grande distribution et ses fournisseurs (2.2.).

\subsection{L'objectivation par la standardisation opérée dans la filière.}

Le cas de l'animal de boucherie présente une particularité vis-à-vis de la marchandisation qui est généralement traitée à partir des notions de désanimalisation et de réification. L'idée est que pour que la chair de l'animal devienne un bien de consommation, il faut la réifier, soit en instaurant une hiérarchie nette entre l'homme et l'animal, soit en occultant son origine animale. L'anthropologie des abattoirs (Vialles, 1987 ; Rémy, 2003) met particulièrement en lumière cette désanimalisation : de fait, ces travaux offrent une représentation de l'activité de l'abattoir centrée sur la dimension éthique du rapport entre homme et animal. Cependant, cette conceptualisation ne permet pas de rendre totalement compte du pro- 
cessus d'objectivation impliqué dans la marchandisation de la viande. En effet, au-delà de cette mise en forme particulière, il existe tout un ensemble de domaines de définition (éthique donc, mais aussi biologique, technique, économique, commercial, etc.) de la production de viande. A partir d'observations participantes dans un abattoir, Séverin Müller (2002) donne à voir comment le travail et la définition de la viande varient : selon le statut et l'occupation du visiteur, c'est le côté spectaculaire de la mise à mort, le suivi des règles sanitaires, le rythme ou la qualité du travail qui est mis en avant. La grande distribution s'intéresse quant à elle à la dimension animale dans la mesure où celle-ci intervient dans la commercialisation de la viande, qui est son objet principal : à ce titre, l'hétérogénéité et l'instabilité biologique de ce produit sont appréhendées comme des obstacles au mode de gestion standardisateur qui est le sien. Nous allons ici nous situer dans cette perspective et retracer à partir des fournisseurs l'important travail de définition du produit, mais avant de s'intéresser plus précisément aux relations entre la grande distribution et ses fournisseurs, il peut être utile d'avoir une vue plus générale de la filière.

Dans le circuit le plus courant, les éleveurs vendent leurs animaux à des coopératives ou à des négociants, qui en rassemblent un certain nombre pour les faire abattre ou les vendre encore vifs à des sociétés de transformation. Ces sociétés achètent donc soit des carcasses soit des animaux qu'elle font abattre pour en récupérer les carcasses, et transforment celles-ci pour les vendre à des bouchers, à des grandes surfaces ou à des entreprises de restauration hors foyer, qui sont en contact avec le client final. Il y a en fait trois transformations possibles. La pre- 
mière consiste à retirer la peau, les viscères, la tête, les pattes, la colonne vertébrale et les abats "à risque" pour aboutir à des demi-carcasses ou à des quartiers. La deuxième transformation consiste à séparer les muscles pour en retirer la membrane, le gras superficiel et les os attenants ; ils sont ensuite regroupés par type de muscle et généralement mis sous vide. On obtient alors de la viande prête à découper $(\mathrm{PAD})^{2}$. La troisième transformation consiste à « piécer » la viande, c'està-dire découper des tranches pour les vendre au détail. Jusqu'aux années 1960, la filière se caractérisait par l'hétérogénéité et la discontinuité des procédures d'évaluation, ainsi que par un manque de transparence, ce qui pénalisait principalement les producteurs et les consommateurs, les carcasses pouvant être réévaluées arbitrairement à la hausse par les intermédiaires. Pour sortir de cette situation, les pouvoirs publics ont initié la mise en place de procédures d'identification et de marquage communes aux différents acteurs de la filière. Ces procédures reposent notamment sur l'utilisation de grilles de classification et de nomenclatures pour le niveau de gras et la conformation des carcasses ${ }^{3}$. Pour la mise en place de ces grilles, le prix des carcasses était enregistré en même temps que leurs principales caractéristiques (l'âge, la race, le sexe, la conformation, l'état d'engraissement, les qualités du muscle et de la graisse) ; la définition des classes de qualité a été réalisée à partir d'une analyse statistique débouchant sur la valeur économique relative de ces caractéristiques (Trift 2005, chap.1). Concernant la définition des morceaux

\footnotetext{
${ }^{2}$ Notons que le degré de transformation ne conditionne pas le type de quantité : les sociétés de transformation peuvent tout aussi bien vendre sous la forme PAD une carcasse entière que du « catégoriel », par exemple une poche de cinq kilos de faux filet : comme ce genre de morceau se vend plus que le reste, les chefs de rayon rachètent du catégoriel de manière à répondre à cette particularité de la demande.

${ }^{3}$ La conformation est la « silhouette » de la carcasse, principalement évaluée sur la proportion et la répartition des muscles. On a d'abord utilisé la grille F.R.A.N.C.E. puis E.U.R.O.P.A. et aujourd'hui S.E.U.R.O.P pour la conformation, et une échelle de 1 à 5 allant du moins au plus gras.
} 
de viande, on retrouve un processus similaire et comme pour les carcasses, les outils d'évaluation ont été produits à partir du traitement de données construites sur les critères d'identification. La mise en place d'une nomenclature unifiée en France est le résultat d'un long processus marqué par les multiples interventions des municipalités pour encadrer et discipliner le commerce de la viande. M. Ferrières (2002) donne de nombreux exemples de ces interventions, et A. Lhuissier décrit la standardisation des transactions au cours de la seconde moitié du XIXème siècle. Partant du constat que le prix de la viande dans les boucheries était hétérogène et trop élevé pour les classes populaires, de nombreuses municipalités ont tenté d'encadrer les pratiques des bouchers en leur imposant des prix maximaux de vente. Ces démarches se sont heurtées à la diversité des pratiques de ventes au détail ${ }^{4}$, et ont ensuite convergé avec les initiatives de boucheries sociétaires (souvent portés par les municipalités) et de vétérinaires pour déboucher sur la diffusion d'une nomenclature des morceaux de viande et de leurs prix relatifs (Lhuissier 2003). On retrouve aujourd'hui ces classifications et cette nomenclature dans le cadre des relations entre la grande distribution et ses fournisseurs, en particulier dans les cahiers des charges où sont spécifiées les caractéristiques du produit et les conditions des transactions. Elles y sont accompagnées de critères supplémentaires, comme des taux de rendement et des proportions, qui permettent à la grande distribution de préciser la définition du produit dans le but d'optimiser sa gestion.

\footnotetext{
${ }^{4}$ Il y avait en effet jusque là trois méthodes principales de formation des prix de la viande : un prix selon l'espèce animale, un prix unique pour toute viande, et un prix selon les morceaux de viande.
} 


\subsection{La définition du produit et des conditions d'échange entre la grande distri- bution et ses fournisseurs.}

Marquant le début de l'intervention directe de la grande distribution, cette étape dans le parcours de la viande est un moment stratégique pour saisir le travail par lequel celle-ci devient une marchandise. Cette transformation nécessite notamment de définir les caractéristiques de chaque carcasse et de fixer les conditions d'échange du produit, qui sont comme nous venons de le voir des questions couplées et classiques de la filière. Lorsque le moniteur viande du groupe enquêté référence un fournisseur, il n'a pas effectué de visite et ne voit pas la viande, car le principal outil de définition du produit est le cahier des charges ${ }^{5}$. Ce dispositif produit une définition particulière de la viande, sous forme de chiffres et de ratios, qui est orientée vers la valorisation du produit. C'est ce que montre entre autres la présence de taux de rendement et la description des conditions commerciales.

On peut être frappé par le contraste entre la concision de la description générale des carcasses, qui comporte au plus quelques indications de préparation ou de découpe, et la précision apportée à leur décomposition. De fait, la sélection des carcasses repose très largement sur le tri qui est effectué par le fournisseur en fonction de classifications et de nomenclatures des morceaux, et s'appuie sur un ensemble restreint de caractéristiques : la classification utilisée, le poids et le $\mathrm{pH}$ de

\footnotetext{
${ }^{5} \mathrm{Il}$ y en a en fait deux, l'un portant sur la première transformation (présentation carcasse) et l'autre sur la seconde transformation (viande $\mathrm{PAD}$, élaborée à partir de carcasses correspondant au premier cahier des charges).
} 
la carcasse, ces informations étant parfois complétées par des indications de couleur et de température de la carcasse, ainsi que d'âge de l'animal à son abattage. Marque de l'importance accordée à la valorisation du produit, la description s'affine ensuite significativement lorsqu'elle désagrège ces carcasses en descendant au niveau des morceaux qui les composent. Sont alors précisés pour chaque morceau, son poids moyen, la proportion qu'il doit représenter par rapport à une bête entière, le taux de rendement minimal qui doit être atteint après travail du morceau en grande surface, et le taux maximal de perte par exsudation. Ces critères de valorisation sont complétés par la définition des relations commerciales avec le fournisseur. Celles-ci spécifient les modalités de commande, de livraison et de fixation des prix, qui sont des éléments intervenant ensuite dans la gestion de l'activité du rayon et notamment dans la fixation du prix de vente au consommateur. Présenté sous la forme d'un prix au kilo ${ }^{6}$, le prix d'achat est établi à plusieurs niveaux et composé de plusieurs strates. Les fournisseurs déterminent un tarif général auquel s'appliquent des remises sur facture, des remises trimestrielles et des remises de fin d'année ; on aboutit ainsi au prix d'achat « trois fois net $»^{7}$. Dans le cadre du référencement, le fournisseur et le moniteur viande négocient pour l'année un tarif général maximal et un taux global de remise ; pendant l'année, ils négocient ensuite chaque semaine des prix maximaux pour les produits, qui sont communiqués sous forme de feuille de cours (autrement dit des mercuriales) aux chefs de rayon, eux-mêmes pouvant ensuite renégocier le prix à la baisse avec les

\footnotetext{
${ }^{6}$ Par exemple, le prix d'une demie carcasse PAD est de $5 € 70$, et celui d'un quartier arrière est de $7 € 80$.

${ }^{7}$ La différence importante entre ces strates est que le prix qui sert de seuil de revente à perte est le tarif général auquel on soustrait les remises sur facture.
} 
fournisseurs. Chaque livraison est accompagnée d'une fiche technique de rendement, qui reprend les ratios et taux de rendement contenus dans les cahiers des charges (dont chaque rayon possède un exemplaire). Cependant, les chefs de rayon utilisent peu cette source d'information, et contrôlent ponctuellement certains lots sur d'autres critères comme le poids de la carcasse, la grosseur du faux filet et du filet, ou bien un aspect fiévreux. Un autre point important sur lequel les fournisseurs sont jugés est la régularité de l'approvisionnement, qui peut avoir des conséquences importantes sur le volume de vente, et qui constitue pour cela une des raisons de changement de fournisseur ; c'est particulièrement net dans le cas des offres promotionnelles, où le fournisseur doit faire des efforts importants à la fois sur les tarifs et sur les quantités.

Comme on le voit ici tant avec le rachat de catégoriel qu'avec la présence de taux de rendement, les modalités d'approvisionnement prennent déjà la marque des spécificités du travail en rayon, et notamment de sa dimension commerciale. Définie par des critères technico-économiques, la viande est présentée dans une mise en forme chiffrée, qui est appropriée aux activités de calcul et de gestion constitutives de sa valorisation. C'est en effet sur le processus de description du produit que se greffent son évaluation et la formation de son prix.

\section{3. « Faire du chiffre » : l'appréciation de la viande par des actes de gestion.}


Lors du passage de la viande dans la grande distribution on assiste en effet, audelà du travail de qualification destiné aux clients, à une autre transformation qui consiste à la représenter sous forme de données chiffrées, qui sont traitées par la grande distribution et pour elle même, dans le cadre de la gestion du rayon. On retrouve sur ce point les caractéristiques dégagées par Jack Goody (1979) : l'écriture et plus généralement la représentation graphique fonctionne comme un « stockage de l'information, qui permet de communiquer à travers le temps et l'espace, et qui fournit à l'homme un procédé de marquage, de mémorisation et d'enregistrement » (p. 145) ; « les énoncés, parce qu'ils sont matérialisés sous forme écrite, peuvent désormais être examinés, manipulés et réordonnés de façon très diverses » (p. 143). Avec la production et le traitement de ces données, la grande distribution prend spécifiquement en charge la question de la valeur du produit et y consacre une part importante de son travail. Une de ses particularités par rapport aux autres forme de distribution est en effet de pousser plus loin la rationalisation du traitement de la valeur, ce qui passe notamment par la mise en rapport de la valorisation du produit et des coût afférents. La grande distribution produit ainsi un travail d'appréciation de la viande en lui attribuant une valeur et un prix. Ce travail repose sur des actes de gestion et des procédures de calcul (Vatin 2007) : l'utilisation d'un certain nombre d'outils transforme la viande et sa circulation en données qui sont ensuite diversement agencées et qui servent de repères pour les stratégies marchandes des chefs de rayon (2.1.) ; en particulier, c'est en rassemblant et en combinant des données représentant respectivement l'approvisionne- 
ment, le fonctionnement du rayon et de l'enseigne, la concurrence et la transaction marchande que le moniteur viande du groupe enquêté élabore les prix (2.2.).

\section{1. la mise en compte de la viande dans le rayon.}

L'activité du rayon est régulée par l'utilisation d'outils qui représentent les flux et les stocks de marchandises sous forme de données, celles-ci étant ensuite diversement combinées pour produire différentes informations. Au-delà de leurs différences d'objet et de temporalité, chacun de ces outils constitue un « dispositif spatial de triage de l'information » (Goody 1979, p. 155) et ouvre par là un espace de calcul (Callon et Muniesa 2003). Parmi ces outils, les cadenciers sont utilisés quotidiennement pour gérer les flux de marchandises, tandis que le compte d'exploitation et le tableau de bord permettent de faire chaque mois un bilan des résultats économiques. En répertoriant respectivement les quantités commandées et les quantités mises en vente dans le rayon libre service, les cadenciers de commande et les cadenciers de coupe facilitent la coordination de l'approvisionnement avec le rythme des ventes. Ils permettent de mesurer les flux de marchandises et de constituer un historique de leurs variations, en fonction duquel les employés du rayon peuvent situer et adapter les pratiques d'achat et de mise en rayon. En limitant les pertes dues à la marchandise qui n'a pu être vendue à temps et le manque à gagner lié à la rupture d'approvisionnement, cette utilisation favorise l'optimisation de la gestion du rayon, qui s'appuient sur des pratiques telles que le rachat de catégoriel : 
Si tu ne veux pas t'embourber dans ton frigo, tu vas commander deux ou trois arrières, et après tu fais du rachat de faux filet. Mais le rachat, tu ne vas pas 1 'acheter à $7 €$, tu vas 1 'acheter à $8 € 40$, pour le vendre à $8 € 90$. Donc total des courses, ça veut dire que tu vas gagner moins d'argent que si tu achetais des arrières. En théorie : parce qu'en pratique si jamais tu achètes tes arrières et que tu es obligé de baisser le prix encore plus pour que ça s'écoule, à un moment donné ça ne sert à rien. Alors il vaut mieux faire du rachat de faux filet, le payer $8 € 40$ et le vendre $8 € 90$; je ne gagnerai pas plus pas moins, mais j'aurai un équilibre matière au fond de mon frigo et une marchandise qui ne va pas restée stockée pendant quinze jours ou trois semaines le temps de l'écouler.

Moniteur viande, février 2005.

Ce raisonnement montre bien comment la valorisation de la viande s'effectue par des actes de gestion, à partir du pratique plus que du «théorique » (selon une formulation récurrente du moniteur viande). L'anticipation du bénéfice attendu n'est pas tant fondée sur un écart de prix entre l'achat et la vente que sur la gestion des caractéristiques de l'approvisionnement, de la transformation et de la commercialisation de la viande. La marchandise peut être fournie à différents stades de transformation et en différents lots (carcasse, quartier, catégoriel), avec un prix d'achat différent selon les cas, tandis les différents morceaux se vendent en plus ou moins grandes quantités, avec des prix et des marges différentes; et l'inadéquation entre ces deux séries de transactions peut occasionner des pertes ou bien un manque à gagner. C'est à partir des critères d'identification de la viande que s'effectue sa mise en chiffres, et c'est en combinant les données ainsi produites que les responsables de rayon procèdent aux calculs qui composent le traitement de la valeur. En effet, au-delà des instruments utilisés quotidiennement, les chefs de rayon élaborent des représentations plus formalisées de l'activité qu'ils utilisent pour sa 
gestion, comme le compte d'exploitation et le tableau de bord. En réunissant et en combinant des données issues de différentes sources, ils établissent un compte d'exploitation et produisent les principaux indicateurs économiques, dont le chiffre d'affaires et le taux de marge ${ }^{8}$. La construction du compte d'exploitation est assez classique, bien que là encore on remarque l'orientation du travail de gestion vers le pratique, puisque les comptes d'exploitation comprennent parfois le calcul de l'économat et de la perte, ces deux éléments venant diminuer la marge « théorique » d'autant ${ }^{9}$. Le fait que cet outil est utilisé au niveau des rayons et à une fréquence importante - et même quotidienne dans certains cas - traduit l'attention accordée à ce mode d'analyse et de gestion de l'activité. Autre outil utilisé dans la gestion de l'activité, le tableau de bord permet quant à lui d'autres calculs, puisqu'il insère les principaux indicateurs économiques dans différentes séries, et les met ainsi en relation avec d'autres chiffres, en particulier les résultats de la période passée et les objectifs fixés pour la période en cours ${ }^{10}$. Il est également utilisé pour comparer les résultats d'un ensemble de rayons boucherie. Ces mises en perspective font l'objet de raffinements divers : calcul des écarts, expression en valeur, en pourcentage et en indice ; distinction des variations de marge nette dues respectivement aux différences de chiffres d'affaire et aux écarts de taux de marge. A côté de ces éléments centraux, d'autres types de tableaux de bord pro-

\footnotetext{
${ }^{8}$ Le chiffre d'affaires est calculé à partir des sorties de caisse, le niveau des stocks à partir d'un inventaire, le montant des achats à partir des factures; et avec ces éléments, on obtient également la marge brute et le taux de marge. Le prix de revient des ventes est calculé en ajoutant le montant des achats à la variation du stock, et la marge brute est constituée par la différence entre le chiffre d'affaires et le prix de revient des ventes. On calcule le taux de marge en rapportant la marge brute au chiffre d'affaires.

${ }^{9}$ L'économat est le coût du matériel d'emballage, et la perte est constituée par la marchandise jetée ou volée.

${ }^{10}$ Ces objectifs sont construits par le moniteur viande à partir de son évaluation des magasins et de ses anticipations, et sont validés par la direction de la branche supermarché.
} 
duisent des indicateurs tels que la proportion du chiffres d'affaires du rayon dans le secteur alimentaire du magasin, la rentabilité horaire des salariés ou la contribution de l'équipe (entendue comme la différence entre la masse de marge et la masse salariale). Ces indicateurs permettent de produire une évaluation des résultats, et cette profusion n'est pas fortuite : la direction des groupes de distribution essaie ainsi de transformer les chefs de rayon et leurs équipes en véritables professionnels du commerce. Ce sont d'ailleurs les bouchers les plus investis dans cette dimension de leur activité, et qui ont par exemple participé à des ouvertures de magasin ou à des redressements de rayons en difficulté, qui deviennent chefs de rayon, et c'est principalement parmi ces derniers que sont choisis les moniteurs techniques.

Il faut qu'ils s'impliquent, c'est des managers hein. Il faut que dans le discours, par rapport à leurs équipes, ils aillent de l'avant. Surtout avec tout ce qui s'est passé en viande depuis dix ans, on a besoin de les rassurer ; parce que c'est vrai que ça a fait devenir frileux. A un moment donné quand vous faisiez du moins 30 ou du moins 40, ça fait chaud dans les compteurs. Il faut les rééduquer à faire du commerce, c'est difficile. Donc on est toujours en train de leur dire il faut mettre des trucs en avant, des promos, des choses, à votre niveau, de façon à faire du chiffre et à les remotiver. (moniteur viande, lui même ancien boucher et chef de rayon, février 2005)

« Faire du chiffre » signifie évidemment réaliser d'importantes ventes et des bénéfices, mais on peut aussi prendre cette expression au pied de la lettre puisque l'activité commerciale s'appuie sur des pratiques de chiffrage et l'utilisation de « compteurs ». C'est avec la production et de la combinaison d'informations de ce type que les acteurs mettent en forme leurs activités et les analysent, puis développent et mettent en œuvre leurs plans d'action, et ceci dans le but d'optimiser la 
valorisation du produit et la gestion du rayon. Les indicateurs produits par le compte d'exploitation et le tableau de bord servent de repères aux chefs de rayon pour développer leur stratégie marchande : l'activité de gestion du rayon guide l'activité commerciale. Les chefs de rayon s'investissent donc dans la comptabilité et la gestion, qui sont des compétences spécifiques, distinctes du métier de boucher proprement dit; il faut donc les « éduquer à faire du commerce ». C'est d'autant plus net dans le groupe enquêté où les chefs de rayon ont pour consigne de relever chaque mois les prix de deux de leurs concurrents directs, et où ils possèdent une assez grande autonomie dans la conduite de leurs affaires : ils peuvent choisir leurs fournisseurs et, tout en restant dans le cadre des accords passés par le moniteur viande, renégocier avec eux les conditions commerciales ; ils peuvent de leur propre chef mettre en place des offres promotionnelles, et modifier à la baisse les prix fixés par le moniteur viande.

\subsection{L'élaboration des prix par la combinaison des comptes.}

L'élaboration des prix est une activité centrale du travail marchand. Dans le groupe enquêté, le moniteur viande fixe les prix de vente aux consommateurs (PVC) en utilisant un outil particulier, la « feuille de découpe », qui rassemble des données issues des différentes étapes du parcours de la viande. En reconstituant cette circulation, il représente également certaines relations entre les acteurs de la filière, et se met ainsi en position d'arbitrer entre leurs objectifs et leurs contraintes. 


\section{Illustration 1 : feuille de découpe.}

Cette feuille de calcul - car c'est à l'origine un fichier Excel ${ }^{\odot}-$ retrace les trois principales étapes d'un caddytainer ${ }^{11}$ de cinq quartiers arrières PAD dans la grande distribution. Le premier tableau de quatre lignes décrit le produit à son arrivée en fonction de son poids, de son prix au kilo, de son prix total. Le tableau qui suit représente la transformation effectuée en rayon, avec la décomposition (la découpe) des arrières en fonction des catégories de morceaux mis en vente. Sont précisés la proportion et le poids de la viande vendue dans chaque catégorie, ainsi que le prix de vente au kilo, ce qui permet au logiciel de calculer le chiffre d'affaires (qui correspond sur cette feuille au « total P.V. ») du à chaque morceau et le chiffre d'affaires total des cinq arrières. La dernière partie de cette feuille en constitue une synthèse, similaire au compte d'exploitation que construit le chef de rayon : elle met en rapport les montants d'achat et de vente pour obtenir la masse et le taux de marge. Pour construire cette feuille, le moniteur viande utilise des éléments provenant des différentes sources déjà identifiées. Le prix d'achat au kilo de la viande PAD provient des feuilles de cours que les fournisseurs font parvenir au moniteur viande chaque semaine ; le poids total du caddytainer, sa répartition en fonction des différents morceaux et le rendement attendu sont des critères définis par le cahier des charges, et des informations qui se trouvent également sur les fiches techniques des caddytainers. Les prix de vente des morceaux ont été fixés par le moniteur viande pour la période précédente. Les taux d'économat et de perte sont des données de marché, c'est-à-dire des niveaux qui sont connus et communs à une

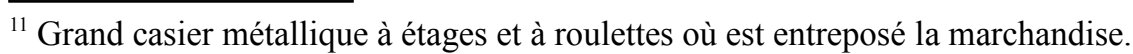


grande partie des acteurs, et que l'on retrouve par exemple dans la presse spécialisée. Ces taux font partie des objectifs que la direction donne aux chefs de rayon.

En réunissant ainsi ces données, cet outil représente les relations entre ces différents éléments et permet de les manipuler. Il met en rapport les objectifs et contraintes propres aux espaces de l'approvisionnement, du rayon, de l'enseigne, de la concurrence et de la transaction finale. Les prix de vente sont également ajustés par comparaison avec les prix pratiqués par les autres enseignes. Le moniteur viande se sert alors des données de marché des listes de prix relevées par les chefs de rayon dans les magasins concurrents. Ces niveaux de prix sont donc des repères pour le moniteur viande, mais leur usage est tempéré par la force de rappel que constitue le calcul de la marge. Le format $\operatorname{Excel}^{\odot}$ a ici son importance. Sur les fiches techniques qu'il récupère de temps à autres sur les caddytainers, le moniteur viande inscrit parfois en face du poids des morceaux son prix de vente, et calcule le chiffre d'affaires et le taux de marge théoriques qui devraient être générés par cette marchandise. Avec la feuille de découpe, il peut effectuer la même prévisualisation, mais aussi et même surtout manipuler cette représentation pour explorer d'autres possibilités, en simulant par exemple des variations de prix d'achat ou de vente, et en observant les conséquences sur les autres grandeurs comme le taux de marge. Cet outil lui permet donc de jouer sur la configuration des relations avec les fournisseurs, l'enseigne et les clients ; en formalisant le fonctionnement pratique de la distribution de la viande, ce dispositif permet d'élaborer les prix à partir de la mise en rapport et de l'arbitrage entre les objectifs et contraintes des 
différents acteurs impliqués dans la commercialisation de la viande : fournisseurs, grande distribution et clients.

La manipulation de ces données permet au moniteur viande de positionner les prix de vente en fonction des taux de marge et chiffres d'affaires attendus, et ce dans le but dans le but d'améliorer la marge. Sur cette feuille de découpe, les achats des clients sont représentés par le prix des produits « P.V.») et le chiffre d'affaires («P.V. TTC »). Les termes par lesquels la grande distribution interprète ici la transaction marchande sont clairement codifiés : il s'agit du taux de marge et du chiffres d'affaires, qui sont en opposition puisque un taux de marge important implique un niveau de prix élevé, ce qui à un certain stade nuit au volume des ventes. L'arbitrage entre ces deux grandeurs se concrétise dans le choix du niveau du prix, qui est lui-même traduit en termes de masse de marge, celle qui sera effectivement obtenue à la fin de la période. A côté de la qualification des produits destinée aux clients, le positionnement du prix constitue donc un autre mode d'action pour la grande distribution : également mobilisé dans le travail de structuration de l'échange, il dessine en creux une figure différente du consommateur. La grande distribution joue en effet beaucoup sur le positionnement des prix pour vendre le produit. Le format $\operatorname{Excel}^{\odot}$ de la feuille de découpe permet au moniteur viande de travailler le positionnement des prix, et cette possibilité est accompagnée d'un second niveau d'ajustement des prix, plus proche du terrain, puisque sous réserve de respecter les prix plafonds, les chefs de rayon du groupe sont invités à jouer sur le prix de vente « pour faire partir le produit au meilleur prix » selon le moniteur viande, qui donne l'exemple du bourguignon qui se vend bien à 
5,55€ dans les Pyrénées-Atlantiques mais qui est positionné à 4,95€ dans les Pyrénées Orientales. Les chefs de rayon doivent même parfois jongler avec les positionnements : dans un magasin enquêté, le chef de rayon mettait en promotion des magrets de canard vendus par lots de trois, car du fait de la mise en place d'une opération promotionnelle nationale de l'enseigne, ils devenaient plus chers au kilo que des magrets vendus à l'unité. Le positionnement du prix fonctionne comme un opérateur d'ajustement entre les échangistes ; d'un côté comme de l'autre, il permet de réduire la transaction à un critère. Le prix fonctionne alors comme un instrument de synthèse des critères de choix, commun au vendeur et à l'acheteur, qui facilite l'accord en le limitant à une dimension, le niveau de prix. Comme le soulignent Michel Callon et Fabian Muniesa (Callon et Muniesa, 2003, p. 213), il s'agit d'un accord sur l'instrument de mesure des valeurs plutôt que d'un accord sur les valeurs. L'expression des ventes en termes de marge et de chiffre d'affaires permet à la grande distribution de traiter ensemble toutes les transactions quels qu'en soient l'objet, comme c'est le cas dans la feuille de découpe, et de les saisir sous l'angle de la masse de marge qu'elles représentent et des niveaux de prix qui y sont associés. Du côté du client, le prix est un des instruments à sa disposition pour le choix des produits, et il peut être mobilisé de différentes manières (Dubuisson-Quellier 2006). Si le client a à sa disposition différents modes de choix et d'action, on retrouve dans le positionnement par le prix une spécification du choix du consommateur qui est proche de celle du rapport qualité-prix : l'examen des qualités, qui peut être plus ou moins largement déployé, est clos pour être replié et situé sur une échelle de prix. Le rapport qualité-prix est ainsi la déclinaison pour 
le client du positionnement du prix par la grande distribution : l'opération par laquelle une multiplicité de critères de choix est ramenée à une seule dimension.

\section{Conclusion.}

Les mécanismes d'identification et d'évaluation des produits constituent l'un des fronts les plus actifs de la sociologie économique, et sont investis par des recherches relevant de divers horizons. Parmi celles-ci, Michel Callon et Fabian Muniesa ont proposé dans un texte à tonalité programmatique d'appréhender les marchés économiques comme des dispositifs de calcul, et Sandrine Barrey a exploré de manière convaincante cette approche dans son analyse du travail de tarification dans un groupe de distribution, en donnant à voir comment la formation des prix repose sur la mobilisation de plusieurs espaces de calcul. Même si son enquête ne permet pas de rendre compte de toutes les opérations effectuées dans le cadre de l'échange marchand, cette approche se révèle pertinente pour la description et la compréhension du travail effectué par les professionnels du marché, qui demeurent malgré tout les acteurs les plus impliqués dans l'organisation de l'activité économique (Cochoy et Dubuisson-Quellier, 2000). Parallèlement ou plutôt perpendiculairement au travail de Sandrine Barrey qui est focalisé sur le niveau général de la grande distribution, suivre un produit particulier le long de son son parcours, comme nous l'avons fait ici pour le cas de la viande, ouvre d'autres prolongements à l'analyse des conditions et des mécanismes de l'échange marchand. Avec une analyse longitudinale de ce type, il devient alors possible de décrire pré- 
cisément le processus de marchandisation qui se déroule tout au long de la circulation du produit. Au-delà de ce premier résultat, l'analyse ouvre également sur une analyse renouvelée de l'articulation entre les mécanismes d'identification et d'évaluation des biens d'une part, et de la formation des prix d'autre part.

Cet angle de vue apporte d'abord un éclairage sur le travail d'objectivation nécessaire à la transformation de la chair animale en produit puis en marchandise, et notamment sur les dispositifs mobilisés. Cela nous a ainsi permis de saisir le travail de transformation et le double traitement que le produit subit : les opérations par lesquelles la viande est objectivée pour devenir un bien sont accompagnées d'autres opérations qui consistent à la mettre en chiffres. La viande devient une marchandise par un processus d'objectivation permettant de faire apparaître des caractéristiques à partir de l'hétérogénéité des carcasses, et ce de manière univoque pour les acteurs, quelles que soient les particularités de ceux-ci. Ce processus simultané d'homogénéisation et de différenciation se déroule avec la circulation de la viande le long de la filière, par la mobilisation de dispositifs métrologiques, de grilles de classification et de nomenclatures ; on retrouve également ces outils dans les relations entre les fournisseurs et la grande distribution, où ils sont complétés par des indications de rendement. La viande est ainsi définie selon des critères technico-économiques et mise en chiffres, ce qui ouvre des possibilités de gestion du produit et de sa circulation. La description de ce processus de marchandisation permet de mettre en lumière une modalité d'articulation entre les processus d'identification et d'évaluation des biens : en étudiant les outils et les opérations mobilisés dans la marchandisation de la viande, on constate que c'est à partir 
des critères et des outils d'objectivation, et par un travail d'extraction et de combinaison de données, que la grande distribution construit l'appréciation des produits. Cette caractéristique est particulièrement manifeste en ce qui concerne l'étape finale de l'élaboration des prix, puisque c'est en rassemblant et en combinant des données représentant respectivement l'approvisionnement, le fonctionnement du rayon et de l'enseigne, la concurrence et la transaction finale que le moniteur viande élabore les prix. Sur ce point, notre analyse complète celle de S. Barrey (2006). La mise en relation des espaces de calcul ne s'effectue pas seulement de manière transversale sur la base des logiques organisationnelles des enseignes et des magasins : elle concerne aussi de manière longitudinale les espaces de la filière de production jusqu'aux clients, dont certaines modalités de choix font partie des modèles d'action de la grande distribution. Nous avons pu décrire dans le détail l'outil et les pratiques par lesquels le moniteur viande (responsable de la tarification pour les produits carnés) relie ces espaces de calcul pour élaborer les prix. La maîtrise de cet outil et des informations qui y sont contenues place l'acteur de la grande distribution dans une position privilégiée d'arbitrage entre les intérêts des différentes parties prenantes impliquées dans la circulation de la viande, les siens y compris.

\section{Références}

Appadurai, A., (Ed.), 1986. The social life of things. Commodities in cultural perspective. Cambridge University Press, Cambridge. 
Barrey, S., 2006. Formation et calcul des prix : le travail de tarification dans la grande distribution. Sociologie du travail 48 (2), 142-158.

Callon, M., Muniesa, F., 2003. Les marchés économiques comme dispositifs collectifs de calcul. Réseaux 122, 191-233.

Callon, M., Meadel, C., Rabeharisoa, V., 2000, L'économie des qualités. Politix 52 (1), 211-239.

Callon, M., (Ed.), 1998. The laws of the Markets. Blackwell, London.

Chantelat, P., 2002. La Nouvelle Sociologie Economique et le lien marchand : des relations personnelles à l'impersonnalité des relations. Revue française de sociologie, 43 (3), 521-556.

Chiffoleau, Y., Laporte, C., 2004. La formation des prix : le marché des vins de Bourgogne. Revue française de sociologie, 45 (4), 653-680.

Cochoy, F., Dubuisson-Quellier, S., 2000. Introduction. Les professionnels du marché : vers une sociologie du travail marchand. Sociologie du travail 42-3, $359-368$. 
Dubuisson-Quellier, S., 2006. De la routine à la délibération. Les arbitrages des consommateurs en situation d'achat. Réseaux 135-136, 253-284.

Eymard-Duvernay, F., 2007. Les institutions de valorisation des biens et du travail. Communication au colloque Théorie de la valeur et sciences sociales, Université Paris X Nanterre.

Eymard-Duvernay, F., 1986. La qualification des produits. In : Salais, R., Thévenot, L. (Ed.), Le travail, marchés, règles et conventions. INSEE - Economica, Paris.

Goody, J., 1979. La raison graphique. Editions de Minuit, Paris.

Lhuissier, A., 2003. Cuts and Classification : the use of nomenclatures as a tool for the reform of the meat trade in France, 1850-1880. Food and Foodways, $n^{\circ} 2$.

Ferrières, M., 2002. Histoire des peurs alimentaires. Le Seuil, Paris.

Müller, S., 2002. Visites à l'abattoir : la mise en scène du travail. Genèses 49, 89109.

Polanyi, K., 1983. La Grande Transformation. Aux origines économiques et politiques de notre temps. Gallimard, Paris. 
Rémy, C., 2003. Une mise à mort industrielle humaine ? L'abattoir ou l'impossible objectivation des animaux. Politix 64.

Sociologie du travail (2002). Dossier débat : la qualité, nº 44, pp. 255-287.

Stanziani, A., 2005. Histoire de la qualité alimentaire. France, XIXe-XXe siècles. Editions du Seuil, Paris.

Stanziani, A., (Ed), 2003. La qualité des produits en France, XVIIIe-XXe siècles. Belin, Paris.

Stanziani, A., 2003 bis. Produits, normes et dynamiques historiques. Sociologie du travail, 45, 259-266.

Testart, A., 2001. Échange marchand, échange non marchand. Revue française de sociologie, 42 (4), 719-748.

Trift, N., 2003. Qualification de l'origine des viandes bovines selon les manières de produire. Thèse en sciences animales de l'INA-PG.

Vatin, F., 2008. L'économie comme acte de gestion. Critique de la définition substantive de l'économie. Sciences de la société 73, 165-184. 
Vatin, F., 1996. Le lait et la raison marchande. Essais de sociologie économique. Presses Universitaires de Rennes, Rennes.

Vialles, N., 1987. Le sang et la chair. Fondation de la Maison des sciences de l'Homme, Paris.

Weber, F., 2000. Transactions marchandes, échanges rituels, relations personnelles. Une ethnographie économique après le Grand Partage. Genèses 41, 85107. 
Illustration 1 : feuille de découpe.

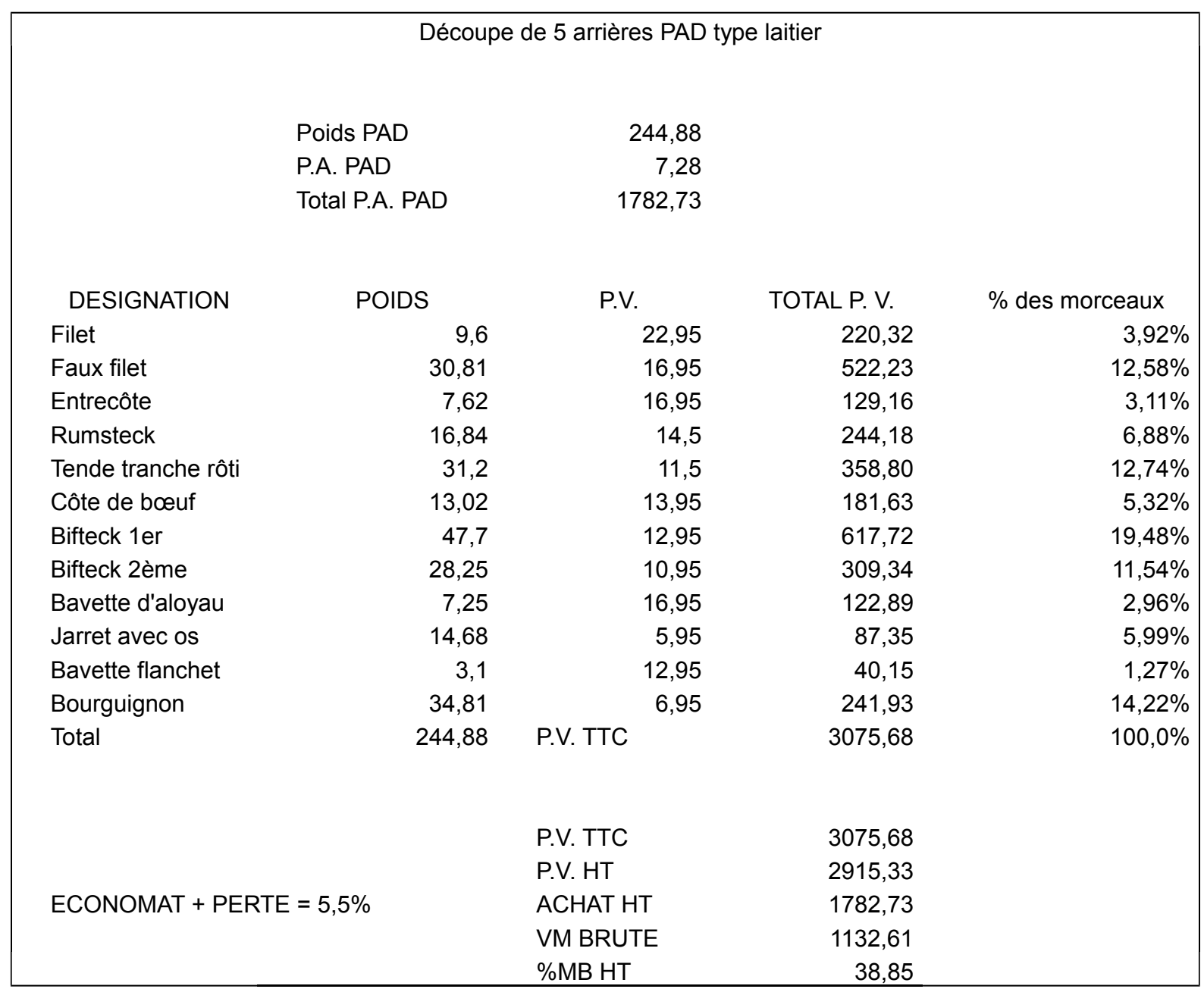

